\title{
The thermodynamics of black holes: from Penrose process to Hawking radiation
}

\author{
Carla Rodrigues Almeida ${ }^{1,2, a}$ \\ 1 Institute for Advanced Studies in Humanities, Essen, Germany \\ 2 Max Planck Research Group Historical Epistemology of the Final Theory Program, Berlin, Germany
}

Received 23 November 2020 / Accepted 14 July 2021 / Published online 5 August 2021

(C) The Author(s), under exclusive licence to EDP Sciences, Springer-Verlag GmbH Germany, part of Springer Nature 2021

\begin{abstract}
In 1969, Roger Penrose proposed a mechanism to extract rotational energy from a Kerr black hole. With this, he inspired two lines of investigation in the years after. On the one side, the Penrose process, as it became known, allowed a comparison between black-hole mechanics and thermodynamics. On the other, it opened a path to a quantum description of those objects. This paper provides a novel take on the events that led to the rise of the thermodynamic theory of black holes, taking as a starting point the Penrose process. It studies the evolution of the research conducted independently by Western and Soviet physicists on the topic, culminating in Stephen Hawking's groundbreaking discovery that black holes should radiate.
\end{abstract}

\section{Introduction}

The formulation of the thermodynamics of black holes is an important development that shaped our current understanding of those objects. Black holes are now understood to have the thermodynamic properties of entropy and temperature and to interact with their environments as a perfect black body, as defined in [40, pp. 12-13]. The identification of black holes as perfect black bodies is, however, a theoretical one and, as such, should be considered critically [18]. A step toward this goal is to analyze the inception of this idea, retracing its origins and evolution to grasp how it was first conceived and the scientific methods employed in this hypothesis.

A black hole was hidden in the first solution of Einstein's equation of general relativity, obtained in 1916. Schwarzschild's Massenpunktes solution [41] described a black hole, but this conclusion was initially rejected. General relativity was in its infancy, and it was broadly misunderstood and underdeveloped [20, p. 255]. The hypothesis that objects with the characteristics of a black hole could exist lacked observational evidence and was too counterintuitive [1]. Theoretical evidence of their existence became strong in the late thirties, indicating that it was a probable consequence of general relativity, but the astrophysics community was still reluctant to accept this result. After the renaissance of Einstein's theory of gravitation in the mid-fifties [9] and the observation of super-dense bodies in the skies at the beginning of the following decade, the situation changed [11]. The rise of relativistic astrophysics and the possibility of identifying quasars as collapsed objects [22, p. 27] renewed interest in the latter, and the sixties became a golden decade for black-hole physics. The concept was improved, new features were unveiled, and black holes were baptized with the name we know them by today.

By the end of the sixties, the main understanding was that black holes were dense astronomical objects with a strong gravitational field wrapped by a fictitious unidirectional membrane, the event horizon, which allowed the entrance but not the escape of matter and radiation from its interior and hid a singularity inside it. A black hole could be described entirely by three classical parameters: mass, charge, and angular momentum. A Schwarzschild black hole has no angular momentum and no charge. Rotating black holes are known as Kerr black holes or, if charged, Kerr-Newman. A rotating black hole would have an exterior region in which the reference frame rotates alongside the black hole, causing an effect called frame-dragging. This region was named ergosphere in $1971 .{ }^{1}$

One influential person who contributed to the development of the concept of black holes during the sixties was Roger Penrose. Among many achievements, he showed that black holes could and would be formed as a consequence of the theory of general relativity [36], and it earned him a Nobel Prize in Physics in 2020 [26]. In 1969, Penrose added another layer to the theory of black holes when he proposed a mechanism to extract energy from a rotating black hole. The Penrose process would happen in the ergosphere, where a particle dropping from infinity would split into two components. One would fall into the black hole, while the other would escape with more mass energy

\footnotetext{
${ }^{\mathrm{a}}$ e-mail: cralmeida00@gmail.com (corresponding author)

1 For a thorough characterization of black holes by the end of the sixties, see [33].
} 
than the original particle had, and thus, the rotational energy of the black hole would be transferred to the motion of this particle outside the ergosphere [38, p. 45]. The energy extraction of a rotating black hole would slow it and decrease its mass, a process that would not be indefinite.

Penrose's mechanism allowed a comparison between black-hole mechanics and thermodynamics - a correlation first accepted as a pure analogy. Penrose's proposal was not the origin of this idea, but its pivotal moment- the missing piece in the puzzle of a consistent formulation of a thermodynamic theory of black holes. One student of John Archibald Wheeler had attempted to develop this theory but could not propose a robust formulation. Progress came after the understanding of Penrose's mechanism and ingenious use of information theory. Meanwhile, the Soviet physicists were looking at the Penrose process through the lenses of quantum field theory, interpreting it as amplification of waves. A complete, semi-consistent description of the thermodynamics of black holes arose from a collaboration between Western and Soviet scientists, partially impaired by the Cold War.

This article puts Penrose's proposal of a mechanism to extract energy from a rotating black hole into a historical context - which does not imply causation, but that had undeniable influence. The focus, however, will be on the ramifications of the formulation of the Penrose process. Starting from its presentation, then to the chain of reasoning that led first to an analogy between black-hole physics and thermodynamics and finally to the theoretical prediction of the Hawking radiation. This last phenomenon convinced the physics community that black holes might be indeed thermodynamical entities. The objective is to shed light on the motivation and methodology that led to this perception. For this, we shall revisit the original papers and analyze technical details to uncover the history they hide.

The history of the quantum phase of research on black holes is briefly outlined in [30], while [8] analyzes the use of information theory to assess the thermodynamical description of those objects. The friendly relationship between Western and Soviet physicists is described in the recollections of physicists who witnessed the events, like Kip Thorne [45] and Igor Novikov [34]. In [31], there is a historical review of the old Soviet academic practices and a glimpse at Yakob Zel'dovich's early career.

The organization of this paper is as follows: Section 2 presents the historical details of the Penrose process, and Sect. 3 retraces the origins of the theory of black-hole thermodynamics. Section 4 focuses on the work of Jacob Bekenstein and his interpretation of this theory, while Sect. 5 explores his formulation of the three laws of black-hole thermodynamics. The criticism of his proposal is presented in Sect. 6, contrasting with the acceptance of an analogy between black-hole mechanics and thermodynamics. Yakob Zel'dovich's views of the Penrose process through the lenses of quantum field theory are outlined in Sect. 7. Finally, Sect. 8 details the combination of Bekenstein's and Zel'dovich's works by Stephen Hawking, ending with his conclusion that black holes should evaporate.

\section{The Penrose process}

The inaugural congress of the European Physical Society — held between 8-12 of April 1969 in Florence, Italy - was a game-changer for the physics of black holes. Themed "The growth points of physics," the package "astronomy, astrophysics, cosmology and relativity" was the first item of concern addressed there [32]. One lecturer in Florence was Roger Penrose, who did an extensive survey on this topic at the conference, exposing the characteristics and key properties known to that date [37]. More than that, he introduced a feature that set the course of research in the following years: a mechanism to extract rotational energy of a Kerr black hole, today known as the Penrose process.

The second half of the twentieth century was also a period for substantial technological progress. During the Cold War, energy consumption increased significantly, and the exploitation of nuclear [43] and fossil energy sources became a vital discussion in the political scenario [39]. Meanwhile, the space race was intensified when, in 1961, the Soviet cosmonaut Yuri Gagarin became the first human to leave the Earth. In 1969, Americans Neil Armstrong and Buzz Aldrin became the first men to step on the Moon [12]. In this context, the Dyson sphere became a symbol for extraterrestrial advanced energy exploitation. Freeman Dyson proposed in 1960 an alternative for scanning radio signals in the search for extraterrestrial intelligent life [19] in the form of detection of infrared radiation. He conceptualized an apparatus that an advanced civilization could use to harvest energy from stars and suggest searching for intelligent life by detecting its characteristic radiation. The Dyson sphere was a habitable thick spherical shell built around a star, with enough machinery to exploit the solar radiation falling onto it from inside.

By the end of his lecture in Florence, Penrose proposed a similar apparatus with a query about energy exploitation. "I want to consider the question of whether it is possible to extract energy out of a 'black hole"" [37, p. 270]. In a thought experiment, he imagined a convoluted system to demonstrate that it was feasible to harvest the energy from a rotating black hole. His first proposal of this apparatus was much like Dyson's: two enormous structures around a black hole built by an advanced civilization, one moving in the direction of rotation, while the other remained still. Penrose gave an intuitive explanation of how it would work (Fig. 1), but he admitted the intricacy 


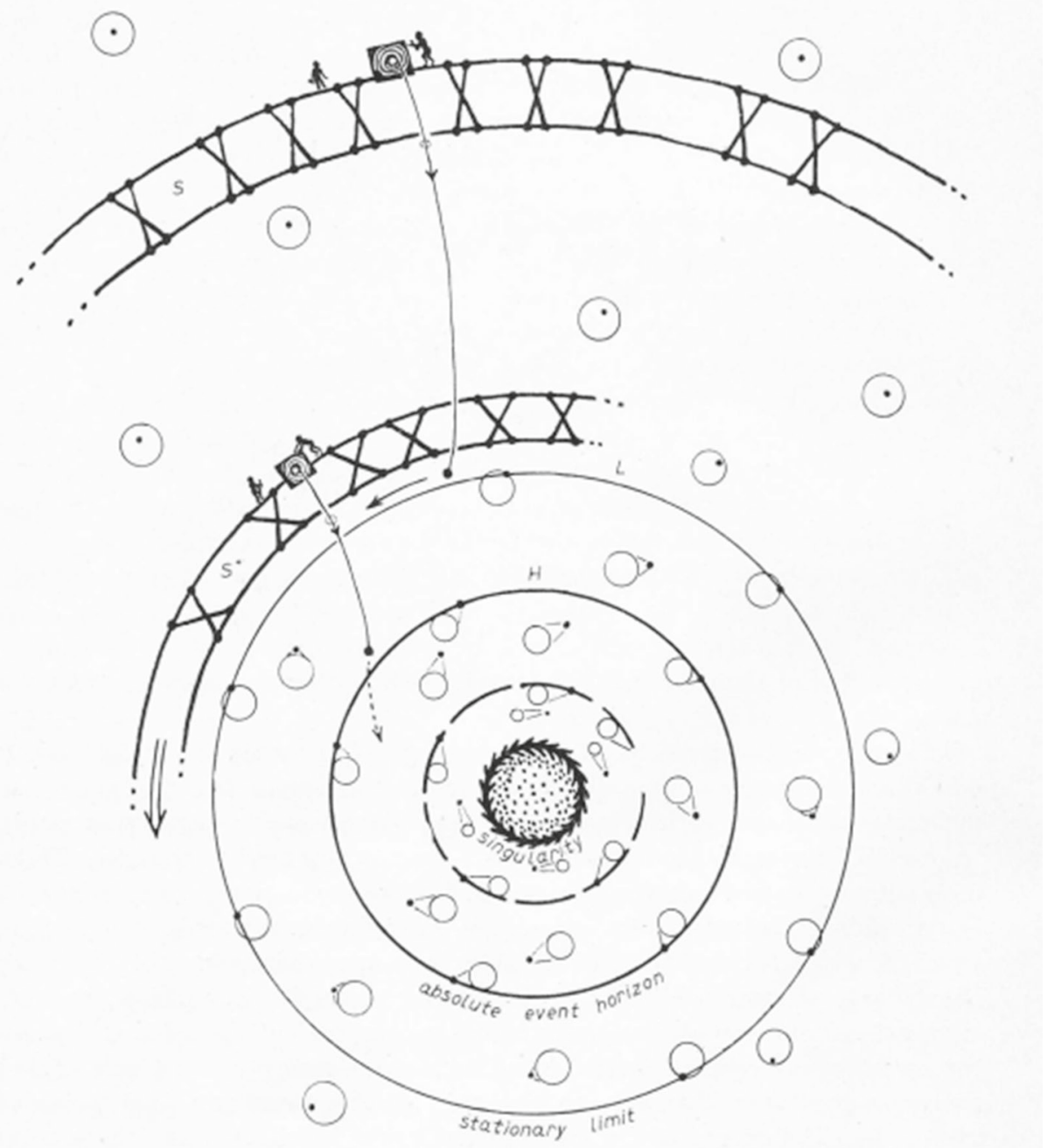

Fig. 5. - Rotating oblack holes (Kerr-Newman solution with $m^{2}>a^{2}+e^{2}$ ). The inhabitants of the structures $S$ and $S^{*}$ are extracting rotational energy from the * black hole $:$

Fig. 1 Extracted from [37, p. 271]. "We imagine a civilization which has built some form of stabilized structure $\mathrm{S}$ surrounding the 'black hole'. If they lower a mass slowly on a (light, inextensible, unbreakable) rope until it reaches $\mathrm{L}$, they will be able to recover, at $\mathrm{S}$, the entire energy content of the mass. If the mass is released as it reaches $\mathrm{L}$ then they will simply have bartered the mass for its energy content. (This is the highest-grade energy, however, namely woundup springs!) But they can do better than this! They also build another structure $\mathrm{S}^{*}$, which rotates, to some extent, with the 'black hole'. The lowering process is continued, using $\mathrm{S}^{*}$, to beyond L. Finally the mass is dropped through $\mathrm{H}$, but in such a way that its energy content, as measured from $\mathrm{S}$, is negative! Thus, the inhabitants of $\mathrm{S}$ are able, in effect, to lower masses into the 'black hole' in such a way that they obtain more than the energy content of the mass. Thus, they extract some of the energy content of the 'black hole' itself in the process. If we examine this in detail, however, we find that the angular momentum of the 'black hole' is also reduced. Thus, in a sense, we have found a way of extracting rotational energy from the 'black hole"'

of his experiment: "Of course, [it] is hardly a practical method! Certain improvements may be possible, e.g., using a ballistic method" [37, p. 272].

The mechanism of the Penrose process depends on the fact that inside the ergosphere, there exist orbits of negative energy when observed from infinity, while the orbits outside this region are of positive energy at infinity. This happens because the metric inside the ergosphere is such that Killing vectors inside it are space-like and those on the outside are time-like. Thus, by lowering an object's orbit from outside to inside the ergosphere, to near the horizon, it can acquire negative energy. The energy it lost could, in theory, be retrieved by the ones who send the object. 


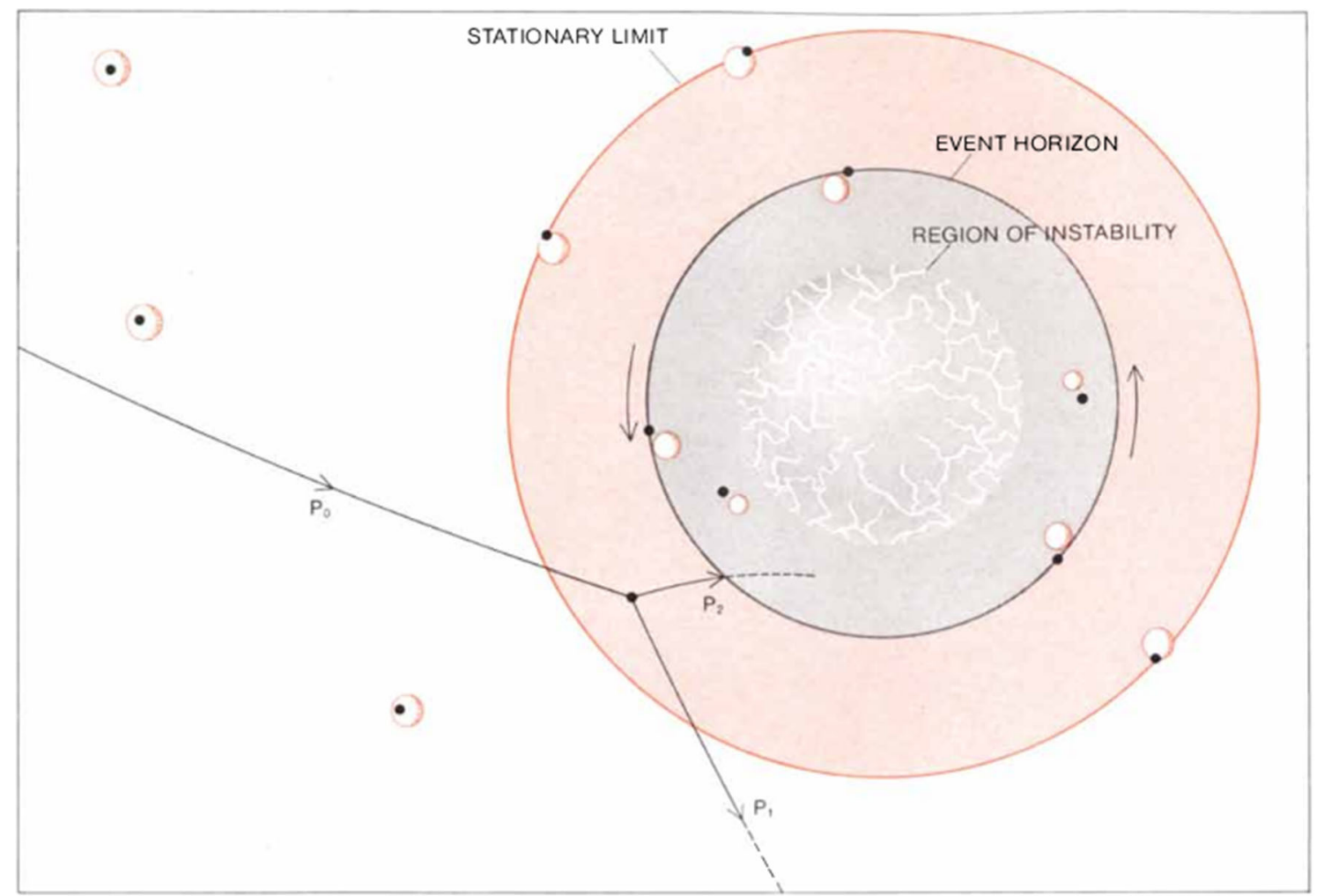

\begin{abstract}
ENERGY CAN BE EXTRACTED from a rotating black hole even though no material object can escape once it has fallen below the event horizon (solid gray). In the case of a rotating black hole the event horizon rotates. The stationary limit is a spheroidal surface (color) on which it is necessary for a signal or a body to travel with the speed of light in order to "stay in the same place" as viewed from infinity. If a particle, $P_{0}$, drops from infinity to within this sur-
\end{abstract}

face, it is possible for it to divide in two in such a way that one component, $P_{2}$, falls into the black hole but the other component, $P_{1}$, escapes back to infinity with more massenergy than the initial particle $P_{0}$. In this way $P_{1}$ extracts some of the rotational energy of the black hole. The location of the internal singularity is uncertain when perturbations in addition to rotation are present, but it is most likely to be along or inside central region of instability.

Fig. 2 Extracted from [38, p. 272]

By the time of publication in a special edition of the Rivista del Nuovo Cimento dedicated to that conference, Penrose had already worked out a ballistic description of the mechanism. The idea revolved around a particle $p_{0}$ dropping from infinity to inside the ergosphere - in Fig. 1, it is the region from $L$ to $H$, in between the absolute event horizon and the sphere of stationary solutions - and nearing the event horizon $H$, "at which point the particle splits into two particles $p_{1}$ and $p_{2}$. The particle $p_{2}$ crosses $H$, but $p_{1}$ escapes $(\ldots)$ possessing more mass-energy content than $p_{0}$ !" [37, p. 272] Throughout the years, Penrose refined the explanation of this phenomenon (Fig. 2).

More than speculation, Penrose aimed to strengthen the search for mechanisms to detect black holes in space by improving the existing theory. He added that its "real significance is to find out what can and what cannot be done in principle since this may have some indirect relevance to astrophysical situations" [37, p. 272]. It indeed led to a theoretical development that Penrose himself could not have foreseen, which changed the perception of those objects. It was a necessary step toward a thermodynamic description of black holes.

\section{Wheeler's boys}

John Archibald Wheeler's interest in black holes started in the late fifties. First a critic of the idea, he grew to become one of the biggest enthusiasts of the concept and later popularized the term "black hole." By the end of the sixties, a conjecture - first proposed and partially proved by physicist Werner Israel in 1967 [27] —intrigued Wheeler. The no-hair theorem, as Wheeler named it, says a black hole can be described by only three classical parameters. As framed in [33, p. 876], "the external gravitational and electromagnetic fields of a stationary black hole (a black hole that has settled down into its 'final' state) are determined uniquely by the hole's mass M, charge $Q$, and intrinsic angular momentum $S$-i.e., the black hole can have no hair (no other independent characteristic)."

Not only was Wheeler a brilliant physicist, but he also became a prime supervisor and educator with a natural charisma for science communication. He used to meet with his research students once a month and regarded them 
as collaborators rather than apprentices. Wheeler often informally suggested various topics of research for them to explore and encouraged their creativity [8]. In 1969, on the project for the research group under his supervision, Wheeler exposed his concerns about black-hole physics [47, p. 28]. As a reaction to the no-hair theorem, one of Wheeler's specific research topics that year was to "[a]nalyze the meaning of 'baryon conservation' and 'lepton conservation' and the principle that 'entropy always increases' in the context of 'black hole physics', where complete collapse washes out all these familiar features of physics" [47, p.30]. According to the no-hair theorem, a black hole has exactly three degrees of freedom. Thus, Wheeler asked how this constraint would fit within the known laws of conservation and thermodynamics.

Wheeler himself did not pursue this thread, however, delegating the task to his students. In his notebooks, he explained why he did not tackle issues like black-hole physics himself. "My reason? Does not reach to the heart of the mystery: pregeometry" [48, p. 17]. At the time, he had a broader research agenda, working on a geometrical description of physics he called pregeometry. In his words, "we'll accept Einstein's general relativity, or 'geometrodynamics' in its standard 1915 form, translated of course into the appropriate quantum version. Second, we accept as tentative working hypothesis the picture of Clifford and Einstein that particles originate from geometry; that there is no such thing as a particle immersed in geometry, but only a particle built out of geometry" $\left[49\right.$, p. 32]. ${ }^{2}$

The first of Wheeler's students to touch on the subject was an undergraduate named Jeffrey M. Greif. In his 1969 Junior thesis Black Holes and Their Entropy, ${ }^{3}$ he proposed a definition for the entropy of a black hole. He suggested it would be the limit of the entropy of a collapsing body right before reaching the singular radius. This definition had many issues. Most noticeable, it did not reconcile with the no-hair theorem. If the entropy depended on the state prior to collapse, the loss in degrees of freedom would be paradoxical. Jacob Bekenstein later noticed that "[Greif] examined the possibility of defining the entropy of a black hole, but lacking many of the recent results in black-hole physics, he did not make a concrete proposal" [5, p. 2333].

One year later, Greek physicist Demetrios Christodoulou took advantage of the most recent tool available at the time to advance one step further in the direction pointed by Greif. Self-taught in physics, Christodoulou got a position as a graduate student in Princeton in 1968 under Wheeler's guidance after finishing high school. In 1970, he published an important report, Reversible and Irreversible Transformations in Black Hole Physics, where he considered the loss of mass of a Kerr black hole in the occurrence of the Penrose process [14]. This extraction of rotational energy, Christodoulou argued, was not indefinite. It would end when the Kerr black hole turned into a Schwarzschild one, that is, when it stopped spinning. Christodoulou explained that losing angular momentum would lead to a decrease in mass and asked which would be the smallest Schwarzschild black hole derived from this process. He named its mass the irreducible mass of the black hole.

Conversely, given a Schwarzschild black hole, one could add rotation by adding massive particles carrying angular momentum. Christodoulou considered this case and the possibility of reversing this action by means of the Penrose process. A Schwarzschild-Kerr-Schwarzschild conversion that results in the original black hole (same mass, no angular momentum) would configure a reversible transformation. Otherwise, it would be an irreversible transformation. For the latter, Christodoulou analyzed that the final Schwarzschild black hole would always have more mass than the first (Fig. 3). Therefore, he concluded the irreducible mass remains constant for reversible transformation, increases for irreversible ones, and never decreases.

Christodoulou remarked that the energy harvesting from a black hole had limited efficacy. In fact, he pointed to the fact that $29 \%$ of the mass of an extreme Kerr black hole could be harvested as energy, "far more energy conversion than in nuclear fission or fusion" $[15$, p. 66]. For a charged black hole, this value can reach up to $50 \%$ $[3$, p. 104]. Christodoulou theorized that the rest would be released as relativistic energy highly ionized, like the relativistic jets escaping from the nuclei of some radio galaxies.

In 1971, Stephen Hawking, investigating the properties of the gravitational radiation generated by the collision of two black holes, discovered that the surface area of the event horizon of the resulting black hole is strictly greater than the sum of their individual areas before they merged [23]. This, he argued, can also be applied for the case of a single black-hole absorbing particles, and thus, he concluded that the surface area of a black hole never decreases. On this subject, Christodoulou remarked in his thesis, "We see, in Hawking's theorem, a strong analogy between the surface area of a black hole and its irreducible mass. Both quantities cannot be reduced. What is the connection between the two quantities?" [15, p. 95]. His answer:

$$
A=16 \pi m_{\text {ir }}^{2},
$$

where $A$ is the surface area of the event horizon, and $m_{\mathrm{ir}}$ is the irreducible mass of the black hole.

2 Wheeler's approach to black holes in the sixties is detailed in [21].

3 Unfortunately, due to limitations caused by the COVID-19 pandemic, this Princeton manuscript was unavailable for external researchers by the time this article was written. The information presented here comes from Jacob Bekenstein's description of Greif's work [3]. They were colleagues working under Wheeler during an overlapping period. 


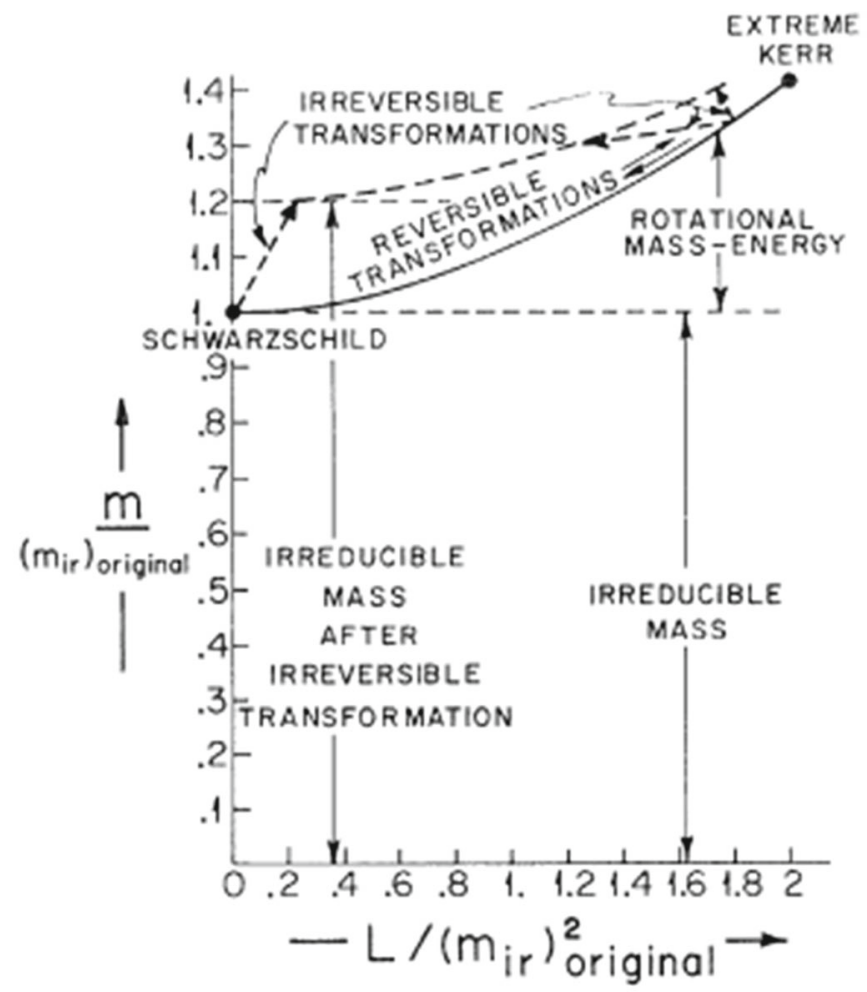

\section{FIG. 1. Mass energy $m$ versus angular momentum $L$ for a black hole of specified irreducible mass $m_{\text {ir }}$ illustrating the difference between reversible trans- formations and irreversible transformations (which increase the irreducible mass).}

Fig. 3 Extracted from [15]

The third of Wheeler's students to contribute to the subject was the young Mexican-Israeli physicist Jacob Bekenstein, who revolutionized the field, giving a new meaning to Christodoulou's result.

\section{A crazy-enough idea}

The connection between black-holes physics and thermodynamics was a seed sprouting in Princeton by the end of the sixties. As mentioned before, Wheeler would often instigate his students to pursue this thread, and to Jacob Bekenstein, he posed an adapted version of an old thermodynamic thought experiment. "What would happen, Wheeler asked Bekenstein, if you mixed hot tea and cold water - thus maximizing the entropy-and poured it into a black hole? Would the entropy simply vanish, pumped out of physical reality? If so, the 'crime' of raising the total entropy would seem to have been pardoned" [8, p. 74]. The beverage, in this case, represents a state of increased entropy in the universe: if it vanished into the event horizon, if the black hole did not have entropy, it would constitute a transgression of the second law of thermodynamics. To find the answer to Wheeler's query, Bekenstein considered a similar contravention in a problem proposed by James Clerk Maxwell in 1867.

The total entropy of an isolated system always increases over time, and it remains constant only for reversible processes. This is the second law of thermodynamics, and Maxwell proposed a situation in which it would not hold. In the nineteenth century, instead of an advanced civilization, Maxwell considered a demon guarding the gate connecting two isolated compartments filled with gas. This demon could open the gate at the right moment to let the more agitated molecules of A enter B and the slower molecules of B enter A. This would cause A to warm up and B to cool down, decreasing its entropy. Heat would flow from the cooler environment to the warmer one, breaking the second law. This thought experiment became known as Maxwell's Demon, and thus, Bekenstein named his supervisor's problem Wheeler's Demon. 
Part of Bekenstein's PhD thesis was devoted to assessing this question. Maxwell's Demon was a well-known controversial problem in physics, and Bekenstein came across an intriguing answer to this paradox in a connection between thermodynamics and information theory. In 1950, Leon Brillouin proposed a solution to this dilemma using information theory [10], which inspired Bekenstein to address Wheeler's Demon in a similar way. Brillouin suggested an increase in entropy relates to loss of information, adopting this relation as a definition for the concept. Bekenstein compared a black hole to a thermodynamic system in equilibrium and argued that, although from the outside only three classical parameters could be measured, from the inside, the other physical features of matter would be preserved. This inaccessibility of information to an exterior observer, this loss in degrees of freedom, would read as the entropy — as defined by Brillouin — of a black hole [5, p. 2336].

According to the theorem by Hawking and Christodoulou, adding mass to a black hole would increase its surface area and decrease the amount of information an observer on the outside has of its interior. Bekenstein argued that the irreducible mass of a black hole would straightforwardly correlate to entropy, according to information theory. The terminology used by Christodoulou, reversible and irreversible transformations, hinted at a connection between his results and the second law of thermodynamics. Bekenstein formalized it: "The irreversibility of an increase in the area of a black hole is reminiscent of the irreversibility of an increase in the entropy of a closed thermodynamic system" [3, p. 104].

Compared to Greif's early attempt, Bekenstein proposed a more concrete definition of the entropy of a black hole. And he availed from the developments not available to Greif at the time, mainly the Penrose process, which led to the Christodoulou-Hawking theorem. Although the correlation between black-holes physics and thermodynamics was welcomed, Bekenstein's approach also received plenty of criticism. He later remarked: "In those days in 1973 when I was often told I was headed the wrong way, I drew some comfort from Wheeler's opinion that 'black holes thermodynamics is crazy, perhaps crazy enough to work'" [7, p. 28].

\section{Bekenstein and the three laws of black-hole thermodynamics}

Following up on Wheeler's thought experiment, Jacob Bekenstein proposed the connection between thermodynamics and black-holes physics in his PhD thesis, Baryon Number, Entropy and Black Hole Physics. He argued this parallelism could help predict "new effects in black holes physics by analogy with familiar results in thermodynamics" [3, p. 107] and exposed the equivalent of the three laws of thermodynamics for this case.

From Christodoulou's equation (1), the entropy of a black hole is proportional to its irreducible mass or equivalently to the area of its event horizon. Thus, Christodoulou-Hawking theorem - the area of a black hole's surface never decreases - reads as the second law.

The first law was not as evident as the second. It is a conservation law that says the energy of an isolated system is constant. As an equation, it translates as

$$
d E=T d S-P d V
$$

where $E, T, S, P$, and $V$ are the energy, temperature, entropy, pressure, and volume of the system, respectively. For a charged, rotating black hole with mass $M$, charge $Q$, and angular momentum $\mathbf{J},{ }^{4}$ Bekenstein considered its rationalized area, ${ }^{5}$

$$
\frac{A}{4 \pi}=2 M\left[M+\left(M^{2}-Q^{2}-a^{2}\right)^{\frac{1}{2}}\right]-Q^{2},
$$

where $a$ is the angular momentum per unit, that is, $\mathbf{a}=\mathbf{J} / M$. The differentiation of Eq. (3) results in,

$$
d M=\frac{\kappa}{2} \frac{d A}{4 \pi}+\boldsymbol{\Omega} \cdot d \mathbf{J}+\Phi d Q
$$

with

$$
\boldsymbol{\Omega}=\frac{4 \pi \mathbf{a}}{A} \quad \text { and } \quad \Phi=\frac{4 \pi Q}{A}\left[M+\left(M^{2}-Q^{2}-a^{2}\right)^{\frac{1}{2}}\right]
$$

\footnotetext{
4 Bekenstein uses $\mathbf{L}$ for the angular momentum. For the sake of clarity, here and throughout the text, a unified notation will be used, overriding the original one.

5 Equation (1) of [23] adapted by Bekenstein to the case of a charged black hole.
} 
Bekesntein concludes that $\boldsymbol{\Omega}$ and $\Phi$ play the role of the rotational angular frequency and electric potential of the black hole, respectively.

Comparing this mass formulae (4) with Eq. (2), Bekenstein identified $A / 4 \pi$ - the square of the irreducible mass $m_{i r}$ from Christodoulou's formulæ Eq. (1) — as entropy and $\kappa / 2$ as temperature. ${ }^{6}$ In Eq. (4), the term $\boldsymbol{\Omega} \cdot d \mathbf{J}+\Phi d Q$ represents the work done on the black hole by an external agent, increasing the angular momentum and charge by $d \mathbf{J}$ and $d Q$, respectively. Thus, it would correspond to $(-P d V)$, which is the work done on a thermodynamic system [5, p. 2335]. With these identifications, the energy content falling into a black hole would be described by parameters depending only on the mass, charge, and angular momentum, in agreement with the no-hair theorem.

The third law came from the identification of $\kappa / 2$ as the temperature of a black hole. For a generalized Kerr black hole, the temperature is always nonnegative, and $\kappa=0$ holds only if the black hole is extreme, that is, if for a given charge and angular momentum, it has minimal mass. Bekenstein considered the process of accretion of selected particles to transform an ordinary black hole into an extreme one. About this transformation, he concluded that "the nearer $[\kappa]$ is to zero (...), the less efficient is further accretion in lowering $[\kappa]$." This, he argued, is equivalent to the third law of thermodynamics, which he phrased as "the nearer a system's temperature $T$ is to absolute zero, the less efficient is any process in lowering $T$ further." And "[t]hus we find an analog to for each of the three laws of thermodynamics in black hole physics!" [3, pp. 106-107].

Coming from Wheeler's thermodynamic thought experiment, Bekenstein questioned whether the correlation between irreducible mass and entropy could be regarded as more than comparison. "Is there a deeper connection between the two subjects? Clearly the concepts of energy and work have similar meanings in thermodynamics and black hole physics. But does the rationalized area of a black hole (the area divided by $16 \pi$ ) have anything to do with entropy?" [3, p. 107]. Bekenstein argued yes, it does. He said, "In everyday physics entropy goes hand in hand with statistics, information, and degradation of energy," via Boltzmann's formulæ for entropy, Brillouin's informational explication, and the introduction of black-hole entropy as a measure of degradation of its energy [3, pp. 129-130]. He proceeded to compare a black hole to a black body. Bekenstein formalized this view in 1973 when he said that "so far the analogies have been of a purely formal nature, primarily because entropy and area have different dimensions" [5, p. 2335]. To obtain a formula for the entropy of a black hole with correct units, his strategy again relied on information theory, identifying information loss as an increase in entropy.

\section{A good analogy}

The correlation between thermodynamics and black-hole physics spiked the curiosity of John M. Bardeen, ${ }^{7}$ Brandon Carter, and Stephen Hawking, who gathered to work on a formulation of their own at the Les Houches Summer School on black holes in 1972 [30] and resulted on a paper published in the following year [2]. In it, they proposed four laws of black-holes mechanics, comparing them with the four laws of thermodynamics. In a more mathematical approach, they explored the geometry of the Kerr solution to calculate the mass and angular momentum in terms of unique time translational and rotational Killing vectors, from where they derived the ensuing laws.

The zeroth law states that the proper acceleration experienced by a test particle near a stationary black hole due to its gravitational pull with a redshift correction, the surface gravity $\kappa$, is constant over the event horizon. This is the same constant $\kappa$ which appears in Eq. (4), ${ }^{8}$ but Bekenstein did not straightforwardly identify it this way, a fact Bardeen, Carter, and Hawking did not fail to notice. "We show that the quantities appearing in (Eq. 4) formulae have well-defined physical interpretation" [2, p. 162].

The other three laws are similar to Bekenstein's. The first law establishes that $\kappa / 8 \pi$ is analogous to the temperature in the same way the area $A$ is analogous to entropy, departing slightly from Bekenstein's definition. The second law refers to the fact that the surface area of each black hole does not decrease with time. And finally, the third law, left unproven, ${ }^{9}$ is the same as the one Bekenstein had already obtained, rewritten as "It is impossible by any procedure, no matter how idealized, to reduce $\kappa$ to zero by a finite sequence of operations" [2, p. 169].

Despite the agreement with Bekenstein's results, Bardeen, Carter, and Hawking could not stress enough that this correlation was nothing but a good analogy. "It should however be emphasized that $(\kappa / 8 \pi)$ and $A$ are distinct from the temperature and entropy of a black hole," otherwise, they argued, it should radiate. "In fact the effective temperature of a black hole is absolute zero. One way of seeing this is to note that a black hole cannot be in equilibrium with black body radiation at any non-zero temperature, because no radiation could be emitted from the hole whereas some radiation would always cross the horizon into the black hole" [2, p. 168]. Their purely geometrical approach to the problem, analyzing a stationary axisymmetric solution of Einstein's equation containing a black hole and surrounded by matter to derive the results, indicates the lack of a deeper connection

${ }^{6}$ In the original notation, $\kappa / 2$ is actually denoted by $\Theta$, and $A / 4 \pi=\alpha$.

7 Son of two-times Nobel laureate John Bardeen.

8 And the reason why this notation was adopted in this text, instead of Bekenstein's $\Theta$.

9 Werner Israel published a proof of this law in 1986 [29]. 
with thermodynamics. At the same time, Bekenstein reasoned outside of a gravitational theory to understand these laws as more than an analogy. In a recollection told to Werner Israel, Hawking admitted that "in writing this paper [with Bardeen and Carter], I was motivated partly by my irritation with Bekenstein who, I felt, had misused my result about increase of the area of the event horizon" [28, p. 264].

Bekenstein did not seem to consider the point raised by Bardeen et al. about the temperature a troublesome issue. In fact, he argued that a black hole could be regarded as a black body cavity with zero temperature. Therefore, Bekenstein agreed that the surface gravity was not to be regarded as the real temperature, calling it a characteristic temperature $[6$, p. 3295]. The increase of the black hole's entropy (its surface area), he argued, would compensate for the loss of thermal entropy [3, p. 128]. If $\kappa / 2$ was not the actual temperature, there should not be any radiation associated with it.

A point Bekenstein perceived as the most serious problem with his view was the violation of the second law of thermodynamics. Ironically, it was the question he was trying to solve in the first place. The black hole's entropy would increase with accretion of matter, causing the entropy of the visible universe to decrease. "It would seem that the second law of thermodynamics is transcendent here in the sense that an exterior observer can never verify by direct measurement that the total entropy of the whole universe does not decrease in the process" [5, p. 2339]. This fault was avoided in Bardeen, Carter, and Hawking's interpretation of this description as an analogy, as the surface area was not actually regarded as entropy. In a follow-up four-page report published in 1972, Black Holes and the Second Law, Bekenstein reformulated this law: "Common entropy plus black-hole entropy never decreases" $[4$, p.738].

Physicist Werner Israel also positioned himself against Bekenstein's views. "Black-hole dynamics has many points of resemblance with thermodynamics. (...) It is perhaps tempting to look for a deeper significance in this parallel. (....) If such a relationship holds it would have far-reaching consequences, for example in cosmology, where it would largely obviate the complications arising from having to treat a universe containing black holes as an open thermodynamical system" [28, p. 267]. Israel argued that Bekenstein could not properly connect entropy influx with energy influx - something Bekenstein had done using information theory - and thus, an increase in the area would not equate to an increase in entropy. Israel proposed that the presence of an external potential energy could disrupt this connection. Bekenstein's use of information theory was innovative and, as such, hard to grasp intuitively. Years later, Israel reflected on his own assessment, saying Bekenstein had "convoluted arguments which, at best, were just short of compelling and, in less fortunate instances, an easy target for criticism" [30, p. 263].

Bekenstein further developed his formulation [6] in the following years. The description of thermodynamics as a statistical discipline and the interpretation of entropy as information loss guided his intuition. Although the proposal was over twenty years old at the time, the use of information theory to assess thermodynamics found one of its first application with this case [8], and this may explain why others were skeptical with the possibility of the thermodynamic description of black holes being more than an analogy.

\section{A different angle}

Since the rise of relativistic astrophysics in the sixties, the Soviet community had been influential in developing black-hole physics. The Cold War pushed a mostly friendly rivalry with Western physicists and highlighted the differences between their scientific backgrounds. In the West, the Penrose process allowed the combination of thermodynamics and black-hole mechanics. Meanwhile, the investigation of this mechanism to extract rotational energy from a Kerr black hole through the lenses of quantum field theory in the Soviet Union led to a wave interpretation of this phenomenon instead of Penrose's ballistic approach.

Self-taught in science, Yakov Borisovich Zel'dovich rose quickly to Academician, the greatest academic position in Soviet Union [34]. He made major contributions to the fields of physical chemistry, nuclear physics, thermodynamics, and cosmology. In 1946, he became the head of the theoretical department of the military project Arzamas-16 and led the research of atomic weapons, working together with Andrei Sakharov on the conception of the Soviet hydrogen bomb [46]. In the sixties, Zel'dovich turned his attention to astrophysics, researching, among other things, the formation of black holes. He suggested, for example, the presence of an accretion disk around massive black holes [50].

As recalled by Kip Thorne [45, p. 428], Zel'dovich learned about the Penrose process from discussions with Thorne himself, Charles Misner, and John Wheeler. In June 1971, Thorne visited Moscow, and he reported one particular interaction with Zel'dovich during that Summer, in which the latter proposed that rotating black holes should radiate. In Zel'dovich's intuition, Thorne explained, the soviet physicist compared a black-hole system to a massive, rotating sphere inside a cylindrical surface. In his analogy, the sphere was the black hole and the interior of the cylinder, the ergosphere. The rotation of the spherical object would reflect and amplify an incident wave, using a fraction of its rotational energy to do so. This would perturb the vacuum fluctuation inside the cylinder and lead to spontaneous radiation. 
Zel'dovich formalized this idea in a short paper published in a Soviet Journal in 1971. Although Thorne credited Zel'dovich's inspiration to be the case of energy extraction of a black hole, the Academician mainly discussed the analogue experiment - the system of plane waves incident on a cylindrical shell with a rotating mass inside it. Zel'dovich concluded that not only the amplification but also the generation of waves would occur. "In the presence of an external reflector with small losses (resonator), the amplification following single scattering may turn into generation." For a black hole described by a Kerr solution, he added that "in a quantum analysis of the wave field one should expect spontaneous radiation of energy and momentum by the rotating body. The effect, however, is negligibly small" [51, pp. 180-181]. Zel'dovich also explained the phenomenon intuitively with a particle interpretation, arguing that the vacuum fluctuation would create a spontaneous pair of particle/anti-particle and the rotation would make this production unstable, and more particles would escape to infinity instead of falling into the horizon. Zel'dovich went beyond the Penrose process and, with a quantum perspective, predicted that rotating black holes should radiate.

The strategy of exploiting analogue experiments to infer properties of an equivalent system is to this date controversial [17], but Zel'dovich had done it before to study stellar configurations. He and Thorne reportedly discussed the physics of rotating starts using a system of a rotating rigid body as an analogue. In 1972, Brandon Carter published a report in favor of this strategy [13], arguing that when applied to the case of black holes, it replicated known features, like the Penrose process and Christodoulou's reversible transformation. In this same year, Zel'dovich released an extended version of his 1971 paper with the title Amplification of cylindrical electromagnetic waves reflected from a rotating body [52]. ${ }^{10}$ This time, he considered the analogy to black holes, but he did not mention, however, the spontaneous radiation again.

Zel'dovich's papers did not generate attention. Even at that year's event dedicated to black holes, the Les Houches Summer School of Theoretical Physics - which some of his associates attended, like Thorne and Soviet astrophysicist Igor Novikov - his results were not mentioned [45, p. 433]. Both of Zel'dovich's papers were published in Soviet journals, which probably contributed to this perceived disinterest. Another reason for this may have to do with Zel'dovich's caution in exploring the phenomenon of energy extraction for the case of black holes, instead, focusing on the analogue systems, refraining from pursuing his bolder assumptions.

In 1973, Alexei Starobinskii, at the time a postdoctoral student of Zel'dovich, revisited his supervisor's result with a more compelling title, Amplification of waves during reflection from a rotating "black hole." In a different manner than Zel'dovich, Starobinskii dealt explicitly with black holes and the incidence of classical waves, properly identifying their amplification as the Penrose process [42]. In September that same year, Stephen Hawking visited Moscow for the first time. In his hotel room, with Zel'dovich and Thorne as witnesses, Starobinskii presented his and his supervisor's results to Hawking, who was unaware of them. To Israel [28, p. 264], Hawking recalled that he liked the idea but was not convinced by the calculations. He mentioned Starobinskii had told him about spontaneous radiation - a phenomenon which Starobinskii himself had not discussed in his paper. Hawking reportedly thought their arguments had physical ground, but he remained skeptical. Inspired by Zel'dovich's and Starobinskii's ideas, Hawking worked on this problem back home. His conclusion, he said, really annoyed him: all black holes must radiate.

\section{The Hawking radiation}

For the case of a Kerr black hole, Starobinskii repeatedly mentioned Zel'dovich's analogue system but avoided his supervisor's quantum approach. Instead, he considered a semiclassical scalar field satisfying a relativistic wave equation - the Klein-Gordon equation. As expected, the amplitude of the reflected wave was greater than of the incident one. It was a description of the Penrose process, Starobinskii argued, without the need to consider composite or unstable particles to work.

After the visit to Moscow, Stephen Hawking had Starobinskii calculations and Zel'dovich's quantum analogue system in his mind. The Soviets convinced him that quantum effects should be considered when studying the evolution of black holes, and Hawking included this premise in a brief report he wrote to Nature [24]. He opened this article titled Black holes explosions?, acknowledging that those effects were usually ignored because near the event horizon of any black hole, the radius of space-time curvature is disproportionally large compared to Planck scale, and thus these contributions were locally small. Nevertheless, he reasoned that "they may still, however, add up to produce a significant effect over the lifetime of the Universe" [24, p. 30].

Hawking adopted a strategy different than Starobinskii's. Whereas the Soviets thought of amplification of waves, Hawking thought of creation and annihilation of particles. He calculated the rate of particle production near the horizon of a Schwarzschild black hole, using simple quantum field theory. He found the number of particles created and emitted to infinity was greater than those annihilated or absorbed, and this difference would amount with time

10 In June 2020, Marion Cromb and collaborators claimed to have shown the effect predicted by Zel'dovich in the analogue system [16]. 
to produce a steady rate of emission. He estimated the emission rate of bosonic particles to be $(\exp (2 \pi \omega / \kappa)-1)^{-1}$, the same expected for a body with temperature in geometrical units of $\kappa / 2 \pi$.

With this, not only rotating black holes would radiate, as suggested by Zel'dovich, but all black holes would. The prediction of the radiation was the last piece of theoretical evidence to convince Hawking that black holes are thermodynamical entities. "Bekenstein suggested on thermodynamic grounds that some multiple of $\kappa$ should be regarded as the temperature of a black hole. He did not, however, suggested that all black hole could emit particles as well as absorb them. For this reason Bardeen, Carter and I considered that the thermodynamical similarity between $\kappa$ and temperature was only an analogy. The present result seems to indicate, however, that there may be more to it than this" [24, p. 31].

The change in status of the thermodynamical analogy of black holes is a key step for understanding its acceptance. This fact is nicely expressed in the words of Kip Thorne, "This [the Hawking radiation] (if Hawking was right) was incontrovertible proof that Bardeen-Carter-Hawking laws of black-hole mechanics are the laws of thermodynamics in disguise, and that, as Bekenstein had claimed two years later, a black hole has an entropy proportional to its surface" [45, p. 436]. The use of analogues to assess different physical processes is a common practice but presents limitations to what kind of information one can extract about the original system. To derive the four laws of black holes as an analogue to thermodynamics was a valid option to study the mechanics of this inaccessible astrophysical system, although it had the constraint of being only an analogy. With the identification of black holes as black bodies, the problem of having to transfer the knowledge from a field to another was solved. ${ }^{11}$

Zel'dovich intuitively predicted that rotating black holes would radiate, and Hawking extended his prediction to all black holes. This difference comes from their approaches to the problem. Zel'dovich's wave description in his analogue system relied on the angular momentum of the massive sphere to destabilize the quantum vacuum fluctuation near the cylindrical shell, here representing the event horizon, causing the emission of particles. Hawking, on the other hand, considered the changing geometry of the space-time near the event horizon instead of the rotation of the black hole to calculate the absorption and emission rates. Hawking was not the first to use quantum field theory in a cosmological scenario. Years before, the American physicist Leonard Parker pioneered [35] investigating particle creation in an expanding universe, but Hawking did not cite him in his papers.

Hawking published an extended version of his report a year later, entitled Particle Creation by Black Holes [25], extending the ideas proposed in the first paper. He reinforced that black holes should radiate with the temperature given by a factor of the surface gravity, and its mass would decrease in the process as the emission rate increased. This, Hawking concluded, meant that black holes not only radiate, but they can also evaporate. He explained, "For a black hole of solar mass $\left(10^{33} \mathrm{~g}\right)$ this temperature is much lower than the $3 \mathrm{~K}$ temperature of the cosmic microwave background. Thus black holes of this size would be absorbing radiation faster than they emitted it and would be increasing in mass. However, in addition to black holes formed by stellar collapse, there might also be much smaller black holes which were formed by density fluctuations in the early universe. These small black holes, being at a higher temperature, would radiate more than they absorbed. (...) When the temperature got up to about $10^{12} \mathrm{~K}$ or when the mass got down to about $10^{14} \mathrm{~g}$ the number of different species of particles being emitted might be so great that the black hole radiated away all its remaining rest mass on a strong interaction time scale of the order of $10^{23}$ s" [25, p. 202].

Despite providing a necessary argument for black holes to be understood as black bodies, using Bekenstein's thermodynamical description and Zel'dovich's quantum approach, Hawking did not solve every problem with this identification. In fact, he created new ones. For example, if a black hole evaporated, its irreducible mass and surface area would decrease, contradicting the theorem that carries his and Christodoulou's name. Hawking explained this by saying there is a constant influx of negative energy into the black hole Christodoulou had not considered, which would constitute a violation of the weak energy condition, ${ }^{12}$ caused by the indeterminacy of particle number and energy density in curved space-time [25, p. 203].

The ad hoc merge between general relativity and quantum field theory is also a point of contention, but Hawking's calculations were solid, and this issue was considered something to be explored instead of being a fundamental crack in the method. Decades later, a debate about the fate of information inside a black hole gained notoriety and exposed another weak spot of the theory. If black holes evaporated, what would happen to the information in their interior? If it also evaporated, it would contradict the postulate that information cannot be lost. This question remains unresolved to this date, and the controversy that ensues it is known as the Black Hole War, thanks to physicists Leonard Susskind and Gerard 't Hooft [44].

\footnotetext{
11 The history of the use of analogue systems in physics is part of the work of Rocco Gaudenzi. I am thankful for our enlightening discussions on this topic.

12 The energy conditions are common assumptions about a generic matter content. In simpler words, the weak energy condition states that the local energy density for any observer is always nonnegative.
} 


\section{Conclusion}

The history of black-holes physics has many pivot points and eventualities that may be a key to understand the scientific practices of highly theoretical areas of knowledge. The development of a thermodynamical theory of black holes, in particular, changed not only our perception of those bodies but also the fields of astrophysics, information theory, and quantum gravity. This paper focused on this first change, outlining the origin of the blackholes thermodynamics idea until the event that solidified this theory as more than a ploy to explore the mechanics of those objects. It identifies the Penrose process as the critical result that allowed the formulation of this thesis and the Hawking radiation as the step to solidify it. This analysis led us full circle around the globe, starting in Europe, with Penrose's lecture and proposal of a mechanism to extract rotational energy of a black hole; then, to the USA, with John Archibald Wheeler's exceptional students; to the Soviet Union, with Zel'dovich's ingenious thinking and interpretation of the Penrose Process; and finally back to Europe, with Hawking's prediction that black holes should radiate and evaporate.

In the sixties, the concept of black holes gained shape with the discovery of more properties, and candidates in the sky made their existence highly probable. In 1969, Roger Penrose compiled this knowledge - that he actively helped to build and for which he won the Nobel Prize in Physics - in a lecture he presented at the inaugural conference of the European Physical Society. In it, he revised and extended the theory of black holes, when he proposed an additional feature, a mechanism to collect rotational energy from a Kerr black hole. He hoped this would lead to new advances in astrophysics and urged the community to search for a black hole in space. The Penrose process proved to be an essential step toward a thermodynamical description of black holes, developed in the following years.

The idea to investigate thermodynamical properties of black holes was a sparkle in John A. Wheeler's mind at that point, and he encouraged his students to pursue it. The first to try was Jeffery M. Greif, who could not make a sound proposal. It was only after the suggestion of the Penrose process that a more robust proposition was possible, and with it, another of Wheeler's students, Demetrios Christodoulou, hinted at a correlation between thermodynamics and black-holes physics. It was Jacob Bekenstein, however, who formalized this connection.

Bekenstein's use of information theory to assess the problem was pioneering. He observed a parallel between the three laws of thermodynamics and some properties of black-holes physics, the second law tied to the Penrose process via Christodoulou's reversible and irreversible transformations. Although Bekenstein's idea was welcomed as a good analogy, he understood it as a genuine connection. He suggested that black holes have a well-defined entropy and are, therefore, thermodynamical entities. This interpretation received many criticisms. Among the critics were John M. Bardeen, Brandon Carter, and Stephen Hawking, who noticed that the temperature in Bekenstein's theory remained ill-defined and that for this connection to be true black holes would have to radiate.

Meanwhile, in the Soviet Union, Yakov B. Zel'dovich, upon learning about the Penrose process, reinterpreted it in a more familiar way to him, using quantum theory. He proposed an analogue experiment that would replicate Penrose's mechanism in a system composed of a massive sphere rotating inside a resonant cylindrical shell. Zel'dovich predicted that Kerr black holes would radiate, although he did not pursue this thread. Alexei Starobinskii extended his supervisor's suggestion focusing primarily on black holes and presented it to Stephen Hawking in 1973. Hawking was intrigued and worked out his own calculations on the subject. To his surprise, he concluded that not only Kerr but all black holes should radiate. More than that, they should eventually evaporate.

The Hawking radiation is the starting point of a new chapter in the history of black-holes physics, the quantum phase of research, as Werner Israel put it [30]. The thermodynamical properties of black holes remain theoretical, but it opened venues and possibilities yet to be explored, hopefully in the near future.

Acknowledgements I wrote this article while I was working at the Max Planck Research Group Historical Epistemology of the Final Theory Program during the COVID-19 pandemic, and I am grateful for the support and encouragement they gave me during this stressful time, in particular the guidance of Alexander Blum and the patience of Kseniia Mohelsky. I edited this paper during my first months as an International Fellow at the Kulturwissenschafliches Institut (KWI), and I appreciate the support of Julika Griem, Sabine Voßkamp, Sebastian Hartwig, and Britta Acksel. I heartily thank Alexander Blum for helping me in every step of research; Stephan Furlan, Rocco Gaudenzi, Gabriela Radulesco for the insightful discussions; and Ricarda Menn for assisting me with proofreading. Also, I offer my deepest gratitude for the suggestions and corrections by the unknown referees. Thank you!

Data Availability Statement This manuscript has associated data in a data repository. [Author's comment: The manuscripts utilized in this research are available at the repository of the Max Planck Institute for the History of Science or Princeton University, except for Wheeler's notebooks. These are publicly available, and there is a link to them at the Reference.] 


\section{References}

1. Almeida, C.R. 2020. Stellar Equilibrium vs. Gravitational Collapse. Eur. Phys. J. H45: 25-48.

2. Bardeen, J.M. et.al. 1973. The Four Laws of Black Hole Mechanics. Commun. Math. Phys. 31: 161-170.

3. Bekenstein, J. 1972a. Baryon Number, Entropy, and Black Holes Physics. Ph.D. Thesis, Princeton University.

4. Bekenstein, J. 1972b. Black Holes and the Second Law. Lett. Nuovo Cimento 4: 737-740.

5. Bekenstein, J. 1973. Black Holes and Entropy. Physical Review D 7 (8), 2333-2346.

6. Bekenstein, J. 1974. Generalized Second Law of Thermodynamcis in Black-hole Physics. Physical Review D 9 (12): 3292-3300.

7. Bekenstein, J. 1980. Black-hole Thermodynamics. Physics Today: 25-31.

8. Belfer, I. 2014. Informing Physics: Jacob Bekenstein and the Informational Turn in Theoretical Physics. Phys. Perspect. 16: $69-97$.

9. Blum, A.S. et al. 2016. The renaissance of general relativity : how and why it happened. Ann. Phys. 528: 344-349.

10. Brillouin, L. 1950. Thermodynamics and Information Theory. American Scientist 38 (4): 594-599.

11. Bonolis, L. 2017. Stellar structure and compact objects before 1940 : Towards relativistic astrophysics. European Physical Journal H. 42: 311-393.

12. Cadbury, D. 2006. Space Race: The Epic Battle Between America and the Soviet Union for Dominion of Space. Harper, New York.

13. Carter, B. 1972. Rigidity of a Black Hole. Nature Physical Science 238: 71-72.

14. Christodoulou, D. 1970. Reversible and Irreversible Transformations in Black Hole Physics. Physical Review Letters 25 (22): 1596-1597.

15. Christodoulou, D. 1971. Investigations in Gravitational Collapse and the Physics of Black Holes. Ph.D. thesis, Princeton University.

16. Cromb, M. et al. 2020. Amplification of Waves from a Rotating Body. Nature Physics 16: 1069-1073.

17. Crowther, K. et al. 2019. What We Cannot Learn from Analogue Experiments. Synthese: 1-26.

18. Curiel, E. 2019. The many definitions of a black hole. Nature Astronomy 3: 27-34.

19. Dyson, F. 1960. Search for Artificial Stellar Sources of Infrared Radiation. Science 131 (3414): 1667-1668.

20. Einsenstaedt, J. 2006. The Curious History of Relativity: How Einstein's Theory of Gravity Was Lost and Found Again. Princeton University Press, Princeton.

21. Furlan, S. 2021. John Wheeler Between Cold Matter and Frozen Stars: The Road towards Black Holes. MPIWG Preprint Series 504

22. Greenstein, J.L. and Schmidt, M. 1964. The Quasi-stellar Radio Sources 3C 48 and 3C 273. The Astrophysical Journal 1: $1-34$.

23. Hawking, S. 1971. Gravitational Radiation from Colliding Black Holes. Physical Review Letters 26 (21): $1344-1346$.

24. Hawking, S. 1974. Black Hole Explosions?. Nature 248: 30-31.

25. Hawking, S. 1975. Particle Creation by Black Holes. Commun. Math. Phys. 43: 199-220.

26. Hill, H.M. 2020. The theory of black hole formation shares the Nobel Prize in Physics. Physics Today 73: 14-17.

27. Israel, W. 1967. Event Horizon in Static Vaccum Space-Times. Physical Review 164 (5): 1776-1779.

28. Israel, W. 1973. Entropy and Black Holes Dynamics. Lett. Nuovo Cimento, 6 (7), 267-269.

29. Israel, W. 1986. Third Law of Black Holes Dynamics: A Formulation and Proof. Physical Review Letters 57 (4): $397-399$.

30. Israel, W. 1987. Dark Stars: the Evolution of an Idea. In Three Hundred Years of Gravitation, eds. Werner Israel; Stephen Hawking. Cambridge, Cambridge University Press: 199-276.

31. Kojevnikov, A.B. 2004. Stalin's Great Science: The Times and Adventures of Soviet Physicists. Imperial College Press, London.

32. Kubbinga, H. 2008. European Physical Society (1968-2008): the Early Years. Europhysics News 39 (1): 16-18.

33. Misner, C et.al. 1973. Gravitation. W. H. Freeman and Company, Princeton.

34. Novikov, I. 1998. The River of Time. Cambridge University Press, Cambridge.

35. Parker, L. 1969. Quantized Fields and Particle Creation in Expanding Universes. I. Physical Review 183 (5): $1057-1068$.

36. Penrose, R. 1965. Gravitational Collapse and Space-Time Singularities. Physical Review Letters 14: 57-59.

37. Penrose, R. 1969. Gravitational Collapse: The Role of General Relativity. Rivista del Nuovo Cimento Serie I, 1: $252-276$.

38. Penrose, R. 1972. Black Holes. Scientific American 226 (5): 38-47.

39. Perović, J. (Ed.). Cold War Energy: A Transnational History of Soviet Oil and Gas. Palgrave Macmillan, London.

40. Planck, M. 1914. The Theory of Heat Radiation. P. Blakiston's Son \& CO. Authorised Translation by M. Masius, Philadelphia. The Project Gutenberg EBook.

41. Schwarzschild, K. 1916. Über das Gravitationsfeld eines Massenpunktes nach der Einsteinschen Theorie. Sitzungsberichte der Königlich Preußischen Akademie der Wissenschaften zu Berlin: 189-196.

42. Starobinskii, A.A. 1973. Amplification of Waves During Reflection from a Rotating "Black Hole". Soviet Physics JETP 64: $48-57$.

43. Strauss, L. 1955. My Faith in the Atomic Future, Reader's Digest, August: 17-21. Last Access in May $17^{\text {th }}$, 2021. (https://energyhistory.yale.edu/library-item/lewis-l-strauss-my-faith-atomic-future-1955)

44. Susskind, L. 2008. Black Hole War: My Battle with Stephen Hawking to Make the World Safe for Quantum Mechanics. Little, Brown and Company, New York.

45. Thorne, K. 1994. Black Holes 83 Time Warps: Einstein's Outrageous Legacy. W.W. Norton Company, New York. 
46. Wellerstein, A.; Geist, E. 2017. The secret of the Soviet hydrogen bomb. Physics Today 70 4: 41-47.

47. Wheeler, J.A. 1969. Relativity Notebook \#16. Last access in May $17^{\text {th }}$, 2021. (https://diglib.amphilsoc.org/islandora/ object/relativity-notebook-16/)

48. Wheeler, J.A. 1970a. Relativity Notebook \#17. Last access in May 17 ${ }^{\text {th }}$, 2021. (https://diglib.amphilsoc.org/islandora/ object/relativity-notebook-17)

49. Wheeler, J.A. 1970b. Particles and Geometry. In Relativity, Springer: 31-42.

50. Zel'dovich, Ya. 1964. The Fate of a Star and the Evolution of Gravitational Energy Upon Accretion. Soviet PhysicsDoklady 9 (3): 195-197.

51. Zel'dovich, Ya.B. 1971. Generation of Waves by a Rotating Body. Journal of Experimental and Theoretical Physics Letters 14: 180-181.

52. Zel'dovich, Ya.B. 1972. Amplification of Cylindrical Electromagnetic Waves Reflected from a Rotating Body. Soviet Physics JETP 35 (6): 1085-1087. 